\title{
The Role of Histology on the Outcome of Sinonasal Carcinomas Treated With Radiation Therapy; a Single Institution Experience
}

\section{Reza Ghalehtaki ( $\square$ rezaght@gmail.com )}

Tehran University of Medical Sciences https://orcid.org/0000-0003-1162-2019

Maryam Taherioun

Tehran University of Medical Sciences

Mahdieh razmkhah

Tehran University of Medical Sciences

\section{Mohammad Shirkhoda}

Tehran University of Medical Sciences

\section{Amirmohsen Jalaeefar}

Tehran University of Medical Sciences

Ata Garajei

Tehran University of Medical Sciences

\section{Mehrdad Jafari}

Tehran University of Medical Sciences

\section{farrokh heidari}

Tehran University of Medical Sciences

Mahdi Aghili

Tehran University of Medical Sciences

Ali Kazemian

Tehran University of Medical Sciences

\section{Research}

Keywords: head and neck cancer, sinonasal cancer, histopathology, gender

Posted Date: September 13th, 2021

DOl: https://doi.org/10.21203/rs.3.rs-861121/v1

License: (c) (1) This work is licensed under a Creative Commons Attribution 4.0 International License. Read Full License 


\section{Abstract}

Background: Malignant epithelial sinonasal tumors are a rare group of head and neck cancers with various histological subtypes that can affect the treatment outcomes in the locally advanced tumors.

Our aim was to investigate the relationship between oncological outcomes and histological type in sinonasal carcinomas.

Material and Method: In this retrospective cohort study we retrieved patients' information from medical archives. Most of patients underwent surgery and all received radiation as indicated, some patients received chemotherapy. We evaluated 3-year overall survival (OS),distant metastases and local recurrence according to histological type.

Results: We studied 28 patients between 2011-2016. Most of them were male. Squamouse cell carcinoma (SCC) was found in 15 (53.5\%), Adenoid cystic carcinoma (AdCC) in 8 (28.5\%), and Adenocarcinoma (AC) in $5(18 \%)$ patients. The highest rate of metastasis was observed in AC. Three-year OS were $48 \%, 50 \%$ and $73 \%$ in SCC, AC and AdCC respectively.

Conclusion: The type of histology affects the oncological outcomes and this relationship can dictate the treatment modality to choose when dealing with malignant sinonasal tumors.

\section{Introduction:}

Sinonasal malignancies are one of the rare cancers of head and neck region and account about $3-5 \%$ of cancers of this anatomical site. The most common histological types are squamous cell carcinoma (SCC) and non-Hodgkin lymphoma (NHL)in the epithelial and non- epithelial tumors, respectively(1). The maxillary antrum is the site that more often affected by this cancer(2). The mean age of individuals who are diagnosed with this cancer is about 62 and men comprise the higher contribution (1).

Management for locally advanced tumors is multimodality treatment and surgery is the main treatment of these tumors. The most important oncologic principle of surgical treatment in sinonasal cancer, with either an open or endoscopic approach, is complete resection with negative margins(3). Today, the use of surgery alone only remains acceptable for early $\mathrm{T} 1$ or $\mathrm{T} 2$ low-grade lesions especially localized to the lower sinonasal cavity. For more advanced lesions, the best option is combined modality therapy(4). For treatment of patients with locally advanced diseases we use definitive concurrent chemoradiation or surgery followed by post-operative radiation with or without chemotherapy in selected patients(5).

Looking at the available literature on this subject, Sanghvi et al analyzed 4,994 patients between 1973 to 2009 and reported the 5,10 and 20 -yearoverall survival to be $52.9 \%, 44.6 \%$ and $29.3 \%$, respectively(2).Phoebe Kuo et al. studied 435 patients between 2004-2012. The 5-year overall survival (OS)in locally advanced cases was $41.5 \%$ and surgery plus chemoradiation had the highest rate of 5 year-survival in comparison with other modalities (6). 
Due to the fact that sinonasal cancers share a relatively small contribution to head and neck cancers as a whole, the accumulation of data especially on various histological subtypes can aid clinicians in better decision making. Various histological types may lead to different oncological outcomes that undermine the importance of histology-based outcome assessment. Here, we aimed to report the role of histology including squamous cell, adenocarcinoma or adenoid cystic carcinoma on the outcome of patients with sinonasal tumors treated with radiation therapy who underwent treatment in our cancer center.

\section{Methods:}

\section{Study design}

In this retrospective cohort study, we evaluated patients with sinonasal cancer between2011 to 2016 at the cancer institute of Iran, affiliated to Tehran University of medical sciences. The records of patients whose disease was proven with pathology were found from the medical archives.

\section{Patients characteristics}

All patients with a history of sinonasal cancer in any sinuses of maxillary, ethmoid, sphenoid and frontal and nasal cavity who received radiation therapy as a part of their treatment were included. The exclusion criteria included lymphoma or sarcoma histology and distant metastases at the time of treatment.

\section{Pretreatment evaluation}

Pre-treatment workup consisted of history taking, physical exam, fiber optic nasal endoscopy, trans-oral or trans-nasal biopsy, magnetic resonance imaging (MRI) or computed tomography (CT) scan of the primary tumor location and neck for evaluating of local and regional staging. The areas of involvement were defined in these work ups. Superstructure extension consisted of involvement of nasal cavity or ethmoid sinus, orbit or pterygopalatine fossa, infratemporal fossa, and through the skull base into the middle cranial fossa. We assessedpulmonary metastases by chest X-ray (CXR) or CT scan at the discretion of physician based on staging.

\section{Treatment description}

\section{Surgery}

Although surgery is the mainstay treatment for sinonasal carcinomas, it is worth noting that obtaining sufficient margins is challenging considering the sophisticated anatomy of the paranasal sinuses. In addition, it is important to recognize when surgical intervention is likely to be impossible. Some causes of inoperability are relatively depending on surgeon's experience, ability to reach free margin, morbidity of procedure, and reconstruction options. In our institute and based on available evidences, anatomic contraindications for open, endoscopic or combined surgeries include patients with gross brain invasion, central skull base or extensive dural invasion, bilateral optic nerve, orbital apex or chiasm infiltration, and cavernous sinus invasion. Extension through the sphenoid sinus walls that usually suggests invasion to the carotid arteries or the cavernous sinus, cutaneous nodularity due to spread of tumor, a significant trismus with gross involvement of the pterygoid and masticatory musculature so that it is not possible to get the 
sufficient margin and also carotid encasement or paravertebral involvement because of lymph node involvement are also considered inoperable cases(7).

\section{Histological examination}

The main point during histological examination by the pathologist is to differentiate among three main histologic types that are SCC, adenoid cystic carcinoma (AdCC) and the adenocarcinoma (AC). Usually these carcinomas are easily diagnosed on hematoxylin and eosin (H\&E) staining with light microscope. For challenging cases that may seldom occur, ancillary studies are needed like immunohistochemistry (IHC) to better define the histological type.

\section{Radiation therapy}

The indications of post-operative radiation were positive or close margin, perineural invasion or lymphovascular invasion and multiple lymph node involvement. For locally advanced patients (T3, T4) we used surgery and adjuvant radiation in operable patients and concurrent chemo-radiation in unresectable or inoperable patients. Due to the challenges described above, the major indication for adjuvant treatment was close or positive or indeterminate surgical margins.

In the adjuvant group, the prescribed dose was 60Gy for negative margins and 66Gyfor positive margins or extra-nodal extension (ENE).For the definitive cases the prescribed dose was 70Gy for the gross tumor and $60 \mathrm{~Gy}$ for areas of suspected microscopic extension and $46 \mathrm{~Gy}$ for elective nodes. Prescribed dose was 46Gyfor cervical elective nodesand 60Gy for positive nodes and 66Gy for those with ENE. The main photon energy was 6MV.

We used our department protocol for 3D conformal radiotherapy for simulation and treatment planning. Information obtained from physical exam, MRI or CT scan and/or surgical pathology report was used for target volume delineation and according to RTOG 91 - 11 protocol. We immobilized patients by thermoplastic mask. The head was made hyperextended for CT simulation and duringtreatment.GTV was defined as all gross disease in physical exam and imaging or any post-op residual disease.CTV was defined as GTV plus $1-1.5 \mathrm{~cm}$ anatomical margin. PTV was defined as CTV plus $0.5 \mathrm{~cm}$ geometrical margin.

\section{Chemotherapy}

For some cases in order to decrease the bulk of tumor and treatment volume we used induction chemotherapy. Induction chemotherapy regimens in advanced disease, were TPF (docetaxel75mg/ $\mathrm{m}^{2} \mathrm{day}$ one and cisplatin $75 \mathrm{mg} / \mathrm{m}^{2}$ day one and $5 F U 750 \mathrm{mg} / \mathrm{m}^{2}$ days $1-5$ continuous IV infusion)or PF (cisplatin $60 \mathrm{mg} / \mathrm{m}^{2}$ day one and 5FU IV infusion $800 \mathrm{mg} / \mathrm{m}^{2}$ days $1-5$ ) every 3 week for $1-3$ cycles. Cisplatin 30-35 $\mathrm{mg} / \mathrm{m}^{2}$ weekly or $100 \mathrm{mg} / \mathrm{m}^{2}$ every 3 weeks were our adjuvant concurrent chemoradiation regimen for those patients with post-op major or minor risk factors. Major risk factors included extra nodal extension or positive surgical margins. Minor risk factors included T3 or T4 primary tumor, perineural invasion(PNI) or lymphovascular invasion( LVI), bone invasion or involvement of lower neck nodes. For definitive cases, all locally advanced tumors underwent concurrent chemoradiation with the same regimens.

\section{Patient evaluation during treatment}


Patients were assessed by radiation oncologists with physical exam and laboratory tests. We evaluated patients every week during treatment and then after radiation for treatment complications.

\section{Post-treatment follow-up}

Three months after treatment we assessed patients by CT scan or MRI at baseline and repeated physical exam every 3-4 month for first two years, every 6 months for the third year and then annually and we used imaging if indicated. For the definitive RT group the interval between follow-up visits were shorter in the first 2 years and then became as the adjuvant group. The first assessment for the definitive group was at 8-12 weeks post treatment to assess the clinical response. The response was defined as the change of primary tumor and involved nodes with respect to pre-treatment images. We used the RECIST 1.0 criteria for response evaluation.

\section{Statistical Analysis}

All eligible patients were recruited consecutively, without sample size and power calculation. The Statistical Package for Social Sciences software (SPSS version 20.0, IBM Corporation, Chicago, IL, US) was utilized to analyze data. To compare the characteristics of various histological types, we used chi squared test. In order to calculate overall survival (OS) and disease-free survival (DFS) rates Kaplan-Meyer survival analysis was opted. The median follow-up durations was calculated with reverse Kaplan-Meyer method for the survivors. The OS was calculated from the beginning of radiation therapy to the date of last follow-up or death. The end-point for DFS was occurrence of locoregional or distant metastasis or death. These rates were presented with $95 \%$ confidence interval (Cl). To determine any significant difference among the histological types in terms of OS and DFS, log-rank tests were used. Level of significance was considered $\mathrm{p}<0.05$.

\section{Results:}

\section{Patients characteristic}

In this study, we analyzed information of 28 patients with sinonasal tumors. Sixty percent of our patients were male. SCC and AC were more common in men with $73 \%$ and $80 \%$, respectively but AdCC was three- fold more common in women. Contribution of desired histological types was as follows: SCC in 15 (53.5\%), AdCC in 8 (28.5\%), and AC in5 (18\%) patients.

The rate of locally advanced cases meaning T3-4 was $43 \%$ inpatients with SCC, 50\% with AdCC and $100 \%$ with AC. The rate of node positive disease was $40 \%$ in patients with SCC, $12.5 \%$ with AdCC and $20 \%$ with AC.

The most common site of tumors was maxillary sinus in 25 patients (92\%). Other sites of primary tumors were ethmoid sinus in 1 (3.5\%) and nasal cavity in 1 (3.5\%) patient. Overall, suprastructure extension including orbital and bony base of skull invasion, pterygoid fossa and cranial fossa invasions were reported in $18 \%$ and $89 \%, 21 \%$ and $7 \%$ respectively (Table 2 ). 
Table 1

$\mathrm{T}$ and $\mathrm{N}$ status of patients based on histological types

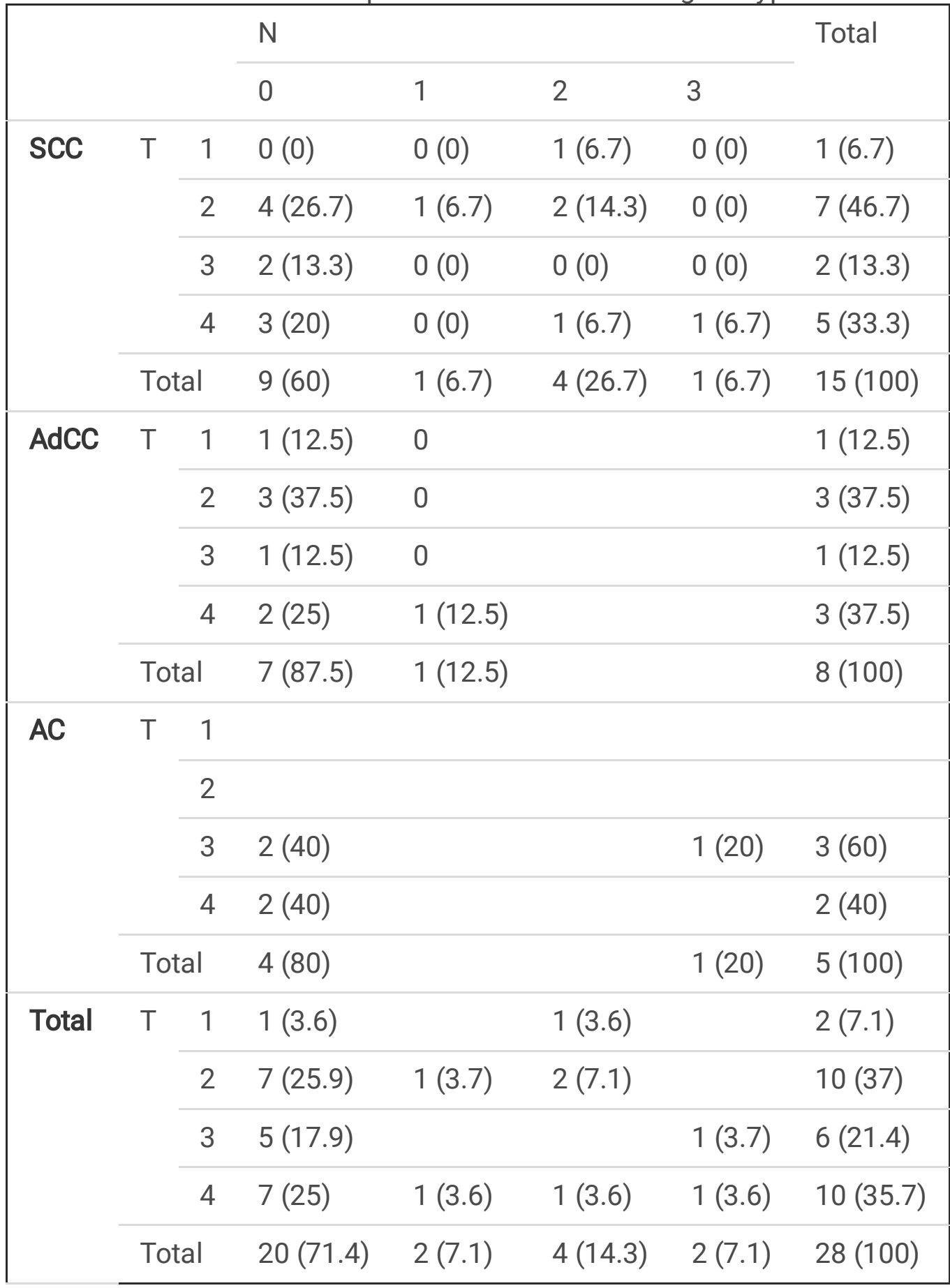


Table 2

Suprastructure extension

\begin{tabular}{|lllll|}
\hline & PFI & OI & CFI & BSI \\
\hline SCC & $6.7 \%$ & $13.3 \%$ & $0 \%$ & $86.7 \%$ \\
\hline AdCC & $50 \%$ & $25 \%$ & $14.3 \%$ & $87.5 \%$ \\
AC & $20 \%$ & $20 \%$ & $20 \%$ & $100 \%$ \\
\hline
\end{tabular}

PFI: Pterygoid fossa invasion, Ol: orbital invasion, CFI: cranial fossa invasion, BI: base of skull bone invasion

\section{Treatment characteristics}

Twenty patients (71.4\%) underwent surgery. According to surgical pathology report, 13 patients had positive margin and 7 patients had negative margins. Among the resected tumors, four had lymphovascular invasion. The rate of LVI was $5 \%, 10 \%$ and $5 \%$ in the SCC, AdCC and AC types, respectively. Eight patients had perineural invasion that was reported in $10 \%, 25 \%$ and $5 \%$ of SCC, AdCC and AC types, respectively.

The setting of radiation therapy was as follows: $25 \%$ definitive, $61 \%$ adjuvant and $14 \%$ salvage. Half of patients underwent radiation therapy to tumor bed as target volume and others received radiation to tumor bed and cervical lymph nodes.

Only 3 patients received induction chemotherapy consisting of TPF regimen before commencement of radiation therapy. Concurrent chemotherapy was prescribed for 11 patients, 8 of them received cisplatin weekly, two received cisplatin every 3 weeks and one patient received cetuximab. Three patients received consolidation chemotherapy after radiation.

\section{Disease outcomes}

In this study we followed patients for a median of 36 months. During this time $3(10.7 \%)$ patients had regional recurrence. Overall, local recurrence was occurred in $4(14.3 \%)$ patients. Nine patients $(32.1 \%)$ suffered from distant metastases in this study. Overall, $44 \%$ of patients not only had distant metastases but also local recurrence. Based on histopathology, $20 \%$ of patients with SCC recurred regionally while none of the patients with $\mathrm{AdCC}$ and $\mathrm{AC}$ had regional recurrence. The rate of local recurrence in patients with SCC,AdCC and ACwas $13.3 \%, 25 \%$ and $0 \%$, respectively. The most common type of histology with distant metastases was AC(40\%). Proportion of distant metastases for SCC and AdCC were $26.7 \%$ and $37.5 \%$, respectively. In metastatic patients with SCC and AC, 50\% had local recurrence too while this proportion for AdCC was $66.5 \%$.

Ten people died during the follow-up duration. The median overall survival in our study was 28,60 and 42 months for SCC, AdCC and AC, respectively. According to our analysis, 3-year overall survival rates was $56 \%$. Patients with SCC,AdCC and AC had 3-year survival rate of $48 \%, 73 \%$, and $50 \%$, respectively. 


\section{Discussion:}

In this study we evaluated 28 patients with sinonasal carcinomas. According to other studies this malignancy is more common among the male that is in line with our finding that men comprised $60 \%$ of patients(1).

Based on the large-scale investigations, the 5 and 10-year survival of malignant sinonasal tumors are $52 \%$ and $44 \%$, respectively(2). Based on our results, the 3 -year survival rate was $56 \%$ that is comparable to other investigations with similar distribution of histological types(8).

Focusing on each histological type, in a study between 1998 to 2012 the 2 and 5-year local recurrence was $37.5 \%$. The involvement of suprastructures decreased the rates of OS,PFS and local control (9).In a study by Michel et al. in 1995-2008 the authors evaluated 33 patients with sinonasal SCC.A quintile was female and the most common site was maxillary sinus. The proportion with invasion to orbital fossa and infra-temporal fossa, base of skull and sphenoid bone were $24 \%, 18 \%, 9 \%$ and $6 \%$, respectively. The 1,5 and 10-year DFS rates were $58 \%, 46 \%$ and $46 \%$, respectively. The 1,5 and 10 -year OS were $70 \%, 40 \%$ and $40 \%$, respectively. Eight patients had recurrence, that 5 of them were local and 3 of them were locoregional. Suprastructure involvement was a significant poor prognostic factor (10). In our study SCC was the most common histopathology with slightly more than half of patients. About two-thirds were male. According to TNM staging, half of them had T2 and most of patients were N0. In terms of suprastructureinvolvement $13 \%$ had orbital invasion and $6.7 \%$ had pterygoid fossa invasion but cranial fossa invasion was not seen in our patients. None of our patients with SCC had regional recurrence but $13 \%$ of them had local recurrence and distant metastasis was seen in $26.7 \%$ of them. In follow up of patients with this pathology 3-year overall survival was $48 \%$ and median survival was 28 months. Our rates are comparable to other studies.

Frequency of AdCC is higher among irradiated sinonasal malignancies than overall population(11).Male and female are equally involved. Perineural, skull base, orbital and cavernous sinus involvements are common in this histopathology. Most of patients die from local recurrence rather than distant metastases. The 5-year OS and DSS in the literature were both $86.5 \%$, and 10-year OS and DSS were both 66\%. 5-year RFS was 71.8\% and was related to the stage of disease. PNI affected the survival and 10-year survival with and without neural infiltration were $44.4 \%$ and $75 \%$, respectively. Percentage of relapse was higher in maxillary sinus, sphenoid sinus and nasopharynx, probably due to relationship with major nerves. Lymph node metastases and distant metastases are rare in this pathology, usually somewhat around 0-5\%and 1-3\%, respectively(12).

In the present study 8 patients had AdCC and about 75\% of them were female. According to TNM staging half of patients presented with T3 or 4 tumors. As we expected, AdCC lymph node involvement was low and $87 \%$ of our patients were neck negative and the remaining had N1 disease. Bone invasion was seen in $87 \%$ of patients that was similar to SCC but suprastructure involvement was higher than SCC that may be justified with the predilection to involve nerves. None of patients had regional recurrence but maximum local recurrence rate was seen in patients with AdCC which has been reported in previous studies using 3D conformal technique for radiation therapy as well(13). According to other studies distant metastases is more commonly seen in this pathology compared to the SCC, and in our analysis $37 \%$ of our patients had distant 
metastases(13). Three-year overall survival and median survival were $73 \%$ and 60 months, respectively. This rate is comparable to other series that reported histology-specific survival rates(11). This pathology had the highest survival rate despite relatively higher metastasis, perhaps due to the low fatality and indolent nature of the metastasis in this type.

Around $10-20 \%$ of primary neoplasms of sinonasal sinus are adenocarcinoma in the radiotherapy cohorts(11).The most common site is ethmoid sinus(40\%). Risk factors include exposure to hardwood dust, nickel and who work with leather, thus, this histopathology is more common in males than females(2 to 6 folds). Due to the anatomical challenges in surgery, local recurrence is the main cause of failure(about $50 \%$, lymph node metastases and distant metastases occur in about $10 \%$ and $13 \%$ of patients, respectively(14).A study was done in 1993-2009 that Bhayani et al evaluated 66 patients with sinonasal AC that half of them received post-operative radiation; 5-year survival, local recurrence and distant metastases were $66 \%, 29 \%$ and $7.6 \%$, respectively. Sphenoid sinus involvement, T4 and higher-grade histology decreased survival significantly(15). In a study carried out between 1995 to 2010 on sinonasal AC, distribution of suprastructure involvement such as skull base and orbit, dura, brain and sphenoid bones were $29 \%$ and $21 \%, 16 \%, 8 \%$ and $8 \%$, respectively. In this study, 1,5 and 10 -year DFS rates were $87 \%, 44 \%$ and $39 \%$,respectively. The 1,5 and 10 year OS rates were $86 \%, 72 \%$ and $50 \%$, respectively(16).

Five patients had AC in our study. The majority were male as was expected due to the relationship between of adenocarcinoma with occupational causative factors. All of patients had locally advanced disease (T3T4) and $80 \%$ were N0.Due to the location of the lesions, advanced T staging and more suprastructure involvement that prohibited curative surgery, there was the highest rate of radical (definitive) irradiation with AC in our study. In patients with AC, despite limitations in surgery, we did not find local or regional recurrence but the highest rate of distant metastases. Three-year overall survival was $50 \%$ and median survival was 42 months. This rate is similar to the SCC type but lower than AdCC due to more aggressive distant metastasis in AC.

Our limitation in this study is the small number of patients and the use of the 3D conformal technique for all patients that limited the prescribed dose for tumors in the challenging locations.

\section{Conclusion:}

The type of histology affects the oncological outcomes and this relationship can dictate the treatment modality to choose when dealing with malignant sinonasal tumors. SCCs need more vigorous local treatment to prevent locoregional recurrences as long as a modest systemic therapy to prevent distant failure. ACs need more emphasis on the systemic therapy to avoid distant metastases as the primary way of treatment failure. Considering very low rates of local or regional failure, seemingly, the radiotherapy alone is a sufficient local treatment for AC. Patients with AdCCs have a long disease course so the care should include quality of life considerations and a robust local treatment whenever possible even in the metastatic cases.

\section{List Of Abbreviations:}


OS: overall survival

SCC: Squamouse cell carcinoma

AdCC: Adenoid cystic carcinoma

AC: Adenocarcinoma

NHL: non-Hodgkin lymphoma

MRI: Magnetic resonance imaging

CT: Computed tomography

CXR: chest X-ray

H\&E: hematoxylin and eosin

IHC: immunohistochemistry

ENE: extra-nodal extension

PNI: perineural invasion

LVI: lymphovascular invasion

DFS: diseaes-free survival

\section{Declarations:}

\section{Ethics approval}

This study was approved by the local institutional review board and the national ethics committee (code:IR.TUMS.IKHC.REC.1399.102).

\section{Consent for publication}

Not applicable

\section{Availability of supporting data}

The data would be available by an eligible request to the corresponding author in terms of complying with copyright laws.

\section{Competing interests}

The authors declare that they have no competing interests

\section{Funding}


This study was supported and funded by the Tehran University of Medical Sciences (TUMS) with Grant number: 99-1-248-47949.

\section{Authors' contributions}

Reza Ghalehtaki Received and designed the analysis, collected data, contributed data or analysis tools, performed the analysis and wrote the paper.

Maryam taherioun Received and designed the analysis, collected data, contributed data or analysis tools, performed the analysis and wrote the paper.

Mahdieh razmkhah Received and designed the analysis, collected data, contributed data or analysis tools, performed the analysis and wrote the paper.

Mohammad Shirkhoda collected data and wrote the paper.

Amirmohsen Jalaeefar collected data and wrote the paper.

Ata Garajei collected data and wrote the paper.

Mehrdad Jafari collected data and wrote the paper.

Farrokh Heidari collected data and wrote the paper.

Mahdi Aghili collected data and wrote the paper.

Ali Kazemian collected data and wrote the paper.

\section{Acknowledgement}

This study was supported and funded by the Tehran University of Medical Sciences (TUMS) with Grant number: 99-1-248-47949..All of the authors declare no industrial link. We thank our colleagues from Department of Radiation Oncology of Cancer Institute, Imam Khomeini Hospital Complex for their contribution in patients' accrual and enrollment. We are deeply grateful of our beloved clerks in Medical Records Archive who helped us a lot during data collection

\section{References:}

1. Kilic S, Shukla PA, Marchiano EJ, Patel RH, Baredes S, Liu JK, et al. Malignant Primary Neoplasms of the Nasal Cavity and Paranasal Sinus [Internet]. Vol. 4, Current Otorhinolaryngology Reports. Springer Science and Business Media B.V.; 2016 [cited 2021 Apr 12]. p. 249-58. Available from: https://link.springer.com/article/10.1007/s40136-016-0134-0

2. Sanghvi S, Khan MN, Patel NR, Yeldandi S, Baredes S, Eloy JA. Epidemiology of sinonasal squamous cell carcinoma: A comprehensive analysis of 4994 patients. Laryngoscope [Internet]. 2014 Jan 1 [cited 2021 Apr 12];124(1):76-83. Available from: https://onlinelibrary.wiley.com/doi/abs/10.1002/lary.24264 
3. Jackson RT, Fitz-Hugh GS, Constable WC. Malignant neoplasms of the nasal cavities and paranasal sinuses: (A retrospective study). Laryngoscope [Internet]. 1977 May 1 [cited 2021 Apr 12];87(5):726-36. Available from: http://doi.wiley.com/10.1002/lary.5540870508

4. Myers LL, Nussenbaum B, Bradford CR, Teknos TN, Esclamado RM, Wolf GT. Paranasal sinus malignancies: An 18-year single institution experience. Laryngoscope [Internet]. 2002 Nov 1 [cited 2021 Apr 12];112(11):1964-9. Available from: https://pubmed.ncbi.nlm.nih.gov/12439163/

5. Bossi P, Farina D, Gatta G, Lombardi D, Nicolai P, Orlandi E. Paranasal sinus cancer [Internet]. Vol. 98, Critical Reviews in Oncology/Hematology. Elsevier Ireland Ltd; 2016 [cited 2021 Apr 12]. p. 45-61. Available from: https://pubmed.ncbi.nlm.nih.gov/26520459/

6. Kuo P, Manes RP, Schwam ZG, Judson BL. Survival Outcomes for Combined Modality Therapy for Sinonasal Undifferentiated Carcinoma. Otolaryngol - Head Neck Surg (United States) [Internet]. 2017 Jan 1 [cited 2021 Apr 12];156(1):132-6. Available from: http://journals.sagepub.com/doi/10.1177/0194599816670146

7. Ganly I, Patel SG, Singh B, Kraus DH, Bridger PG, Cantu G, et al. Craniofacial resection for malignant paranasal sinus tumors: Report of an international collaborative study. Head Neck [Internet]. $2005 \mathrm{Jul}$ [cited 2021 Apr 12];27(7):575-84. Available from: https://pubmed.ncbi.nlm.nih.gov/15825201/

8. Esassolak M, Rhinology YA-, 2003 undefined. Radiotherapy in maxillary sinus carcinomas: evaluation of 79 cases. academia.edu [Internet]. [cited 2021 Aug 25]; Available from: https://www.academia.edu/download/41830671/Radiotherapy_in_maxillary_sinus_carcinom201601317671-7hookm.pdf

9. Paré A, Blanchard P, Rosellini S, Aupérin A, Gorphe P, Casiraghi O, et al. Outcomes of multimodal management for sinonasal squamous cell carcinoma. J Cranio-Maxillofacial Surg. 2017 Aug 1;45(8):1124-32.

10. Michel J, Fakhry N, Mancini J, Braustein D, Moreddu E, Giovanni A, et al. Sinonasal squamous cell carcinomas: Clinical outcomes and predictive factors. Int J Oral Maxillofac Surg. 2014 Jan 1;43(1):1-6.

11. Askoxylakis V, Hegenbarth P, Timke C, Saleh-Ebrahimi L, Debus J, Röder F, et al. Intensity modulated radiation therapy (IMRT) for sinonasal tumors: A single center long-term clinical analysis. Radiat Oncol. 2016 Feb 4;11(1).

12. Volpi L, Bignami M, Lepera D, Karligkiotis A, Pistochini A, Ottini G, et al. Endoscopic endonasal resection of adenoid cystic carcinoma of the sinonasal tract and skull base. Laryngoscope [Internet]. 2019 May 1 [cited 2021 Apr 13];129(5):1071-7. Available from: https://pubmed.ncbi.nlm.nih.gov/30450555/

13. Hoppe B, Stegman L, ... MZ-IJ of, 2007 undefined. Treatment of nasal cavity and paranasal sinus cancer with modern radiotherapy techniques in the postoperative setting-the MSKCC experience. Elsevier [Internet]. [cited 2021 Aug 12]; Available from: https://www.sciencedirect.com/science/article/pii/S0360301606029993? casa_token=aheWLUeYiilAAAAA:73BzXIL72vE4M-SkORMPMZQ9NHRAg8vouBZ2P79IwFutmDuH4KqvDXLkiK8NH66al6yXTMT0Q

14. Lund VJ, Chisholm EJ, Takes RP, Suárez C, Mendenhall WM, Rinaldo A, et al. Evidence for treatment strategies in sinonasal adenocarcinoma. Head Neck [Internet]. 2012 Aug 1 [cited 2021 Apr

Page 12/13 
13];34(8):1168-78. Available from: http://doi.wiley.com/10.1002/hed.21770

15. Bhayani MK, Yilmaz T, Sweeney A, Calzada G, Roberts DB, Levine NB, et al. Sinonasal adenocarcinoma: A 16-year experience at a single institution. In: Head and Neck [Internet]. John Wiley and Sons Inc.; 2014 [cited 2021 Apr 13]. p. 1490-6. Available from: https://pubmed.ncbi.nlm.nih.gov/23996784/

16. Michel J, Radulesco T, Penicaud M, Mancini J, Dessi P. Sinonasal adenocarcinoma: clinical outcomes and predictive factors. Int J Oral Maxillofac Surg. 2017 Apr 1;46(4):422-7.

\section{Figures}

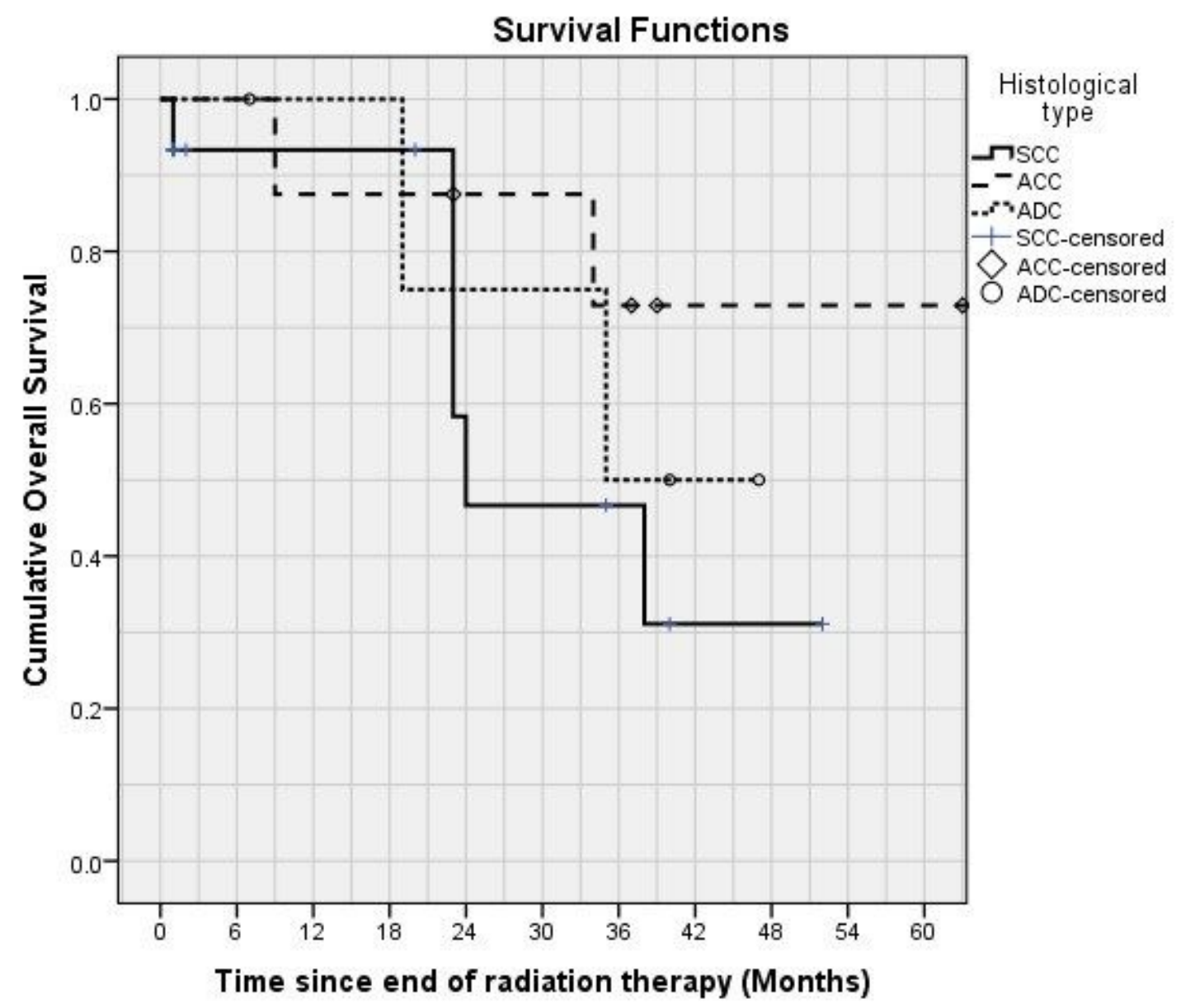

Figure 1

The Kaplan-Meyer survival curve based on the histological subtypes. 